\title{
HSV-1 miR-H6 Inhibits HSV-1 Replication and IL-6 Expression in Human Corneal Epithelial Cells In Vitro
}

\author{
Fang Duan, ${ }^{1}$ Jingyu Liao, ${ }^{1}$ Qiang Huang, ${ }^{1}$ Yuhong Nie, ${ }^{1,2}$ and Kaili Wu ${ }^{1}$ \\ ${ }^{1}$ Zhongshan Ophthalmic Center, State Key Laboratory of Ophthalmology, Sun Yat-sen University, 54 Xianlie Road, \\ Guangzhou 510060, China \\ ${ }^{2}$ Department of Ophthalmology, Renmin Hospital, Wuhan University, Wuhan 430060, China
}

Correspondence should be addressed to Kaili Wu, wukaili@mail.sysu.edu.cn

Received 18 December 2011; Accepted 1 February 2012

Academic Editor: Lbachir BenMohamed

Copyright () 2012 Fang Duan et al. This is an open access article distributed under the Creative Commons Attribution License, which permits unrestricted use, distribution, and reproduction in any medium, provided the original work is properly cited.

\begin{abstract}
HSV-1 infection in the cornea could lead to blindness. The infected cell polypeptide 4 (ICP4) of herpes simplex virus 1 (HSV-1) is a regulator of viral transcription that is required for productive infection. It has been previously demonstrated that miR-H6 encoded from HSV-1 genome targets ICP4 to help maintain latency. In this study, synthesized miR-H6 mimics were transfected into HSV-1-infected human cornea epithelial (HCE) cells. The inhibition of HSV-1 replication and viral ICP4 expression in miRH6-transfected HCE was confirmed by plaque assay, immunofluorescence, and Western blot. Compared to nontransfection or mock, miR-H6 produced a low-titer HSV-1 and weak ICP4 expression. In addition, miR-H6 can decrease the interleukin 6 released into the medium, which was determined by ELISA. Taken together, the data suggests that miR-H6 targeting of ICP4 inhibits HSV-1 productive infection and decreases interleukin 6 production in HCE, and this may provide an approach to prevent HSV-1 lytic infection and inhibit corneal inflammation.
\end{abstract}

\section{Introduction}

Herpes simplex virus type 1 (HSV-1) is a linear doublestranded DNA virus that mainly infects epithelial and neuronal cells. The productive infection of HSV-1 entails a cascade of gene expression (immediate-early, early, and late genes), viral DNA replication, assembly and egress of virus. The immediate-early (IE) gene ICP4 (infected-cell polypeptide 4) of HSV-1 is one of the major regulatory genes required for efficient transcription of early and late viral genes and drives HSV-1 through the productive replication cycle $[1,2]$. ICP4 also plays a role in reactivation of HSV-1 from latency. The regulation of ICP4 seems to be exerted at the posttranscriptional level by the latency-associated transcripts (LATs) $[3,4]$. Inhibition of ICP4 gene can suppress viral production in various cells [5-8].

MicroRNAs (miRNAs) are small, 21 23 nucleotide noncoding RNAs that play an important role in the post-transcriptional regulation of gene expression in a wide range of organisms from unicellular eukaryotes to multicellular eukaryotes by a variety of mechanisms [9]. The existence of viral miRNAs was first reported in Epstein-Barr virus in 2004 [10].
The discovery of miRNAs encoded by viruses suggests that viruses have evolved to exploit RNA silencing for regulation of their own genes, host genes, or both and contribute to the functions including (I) latent and lytic viral infection, (II) immune evasion, (III) prevention of apoptosis, (IV) viral replication, and (V) others [11-13]. As for HSV-1, the first microRNA, miR-H1, expressed as a late gene in productive infection, was reported in 2006 [14]. Also, HSV-1 infection of human brain cells inducing a miRNA-146a that mediates inflammatory signaling was firstly reported in 2009 [15]. Up to now, 16 HSV-1 miRNAs have been found $[4,12,14,16]$. Of them, both miR-H1 and miR-H6 are located upstream to the LAT promoter. miR-H1 is encoded by sequences upstream of the LAT promoter in the LAT sense direction, while miR-H6 is located in an LAT antisense direction [16]. Umbach et al. first found that miR-H6 downregulates the expression of ICP4 proteins, suggesting a contribution to the establishment and maintenance of viral latency [4]. Similarly, the latent infection is characterized by the abundantly expressed locus that encodes the LAT, which can repress lytic replication and IE gene expression in a neuronal cell line [3]. However, miR-H6 and H1, detected as early as 
$2 \mathrm{hr}$ postinfection (p.i.) in cultured cells, were abundantly expressed in the HSV-1 productive infection sample, whereas the other HSV-1 miRNAs (i.e., miR-H2, -H3, and -H4) were greatly produced during the latent infection $[16,17]$. Meanwhile, miR-H6 expression was significantly reduced in cells infected with a mutant HSV-2 virus with an insertion of a sequence between the LAT promoter and miR-H6 [18], and also in latently, but not in acutely, infected mouse ganglia with HSV-1 LAT deletion mutants (dlLAT1.8) [17]. These results suggested that when miR-H6 is expressed abundantly during lytic infection, it may play other roles during viral infection besides establishing and maintaining the latent infection $[12,17]$.

As a major pathogen to human beings, HSV-1 can cause a variety of diseases and can even be life threatening $[19,20]$. Common ocular manifestations of HSV-1 infection include blepharitis, conjunctivitis, keratitis, and uveitis; of them, recurrent viral keratitis can lead to severe corneal blindness [2]. In addition, the cornea tissue was suggested to be the site where HSV-1 can establish latent infection $[2,21,22]$. The corneal epithelium is composed of several layers of cells in front of the stroma. Thus, it is given the important role of protecting from the invasion of exogenous viruses. The primary infection of HSV-1 in corneal epithelial cells is typically displayed by dendritic lesions. However, HSV-1induced keratitis also frequently appeared, as the immunopathology of inflammation was not directly produced by a great number of lytic infection of virus [23-26]. In such cases, cytokines play important roles in the virus-induced immunopathology. One cytokine known to contribute to immune response to HSV-1 is interleukin-6 (IL-6), which is a cytokine with pleiotropic activities, including both proinflammatory and anti-inflammatory activities [27]. Meanwhile, within the LAT and ICP0 promoter of HSV-1, there are IL- 6 response elements, possible binding sites of the IL-6-induced transcription factors. Viral constructs with deletion of the IL-6 response element in the LAT promoter reactivate at much lower rates than similar constructs without the deletion [28]. IL- 6 induced by HSV-1 infection has been reported in various cells such as leukocytes [29], epithelial cell EMT6 , and $\mathrm{HaCat}[30,31]$, as well as cornea epithelial cells and fibroblasts [32].

Regarding the abundant expression in lytic infection and the location of miR-H6 encoding sequence (upstream of LAT promoter), we wonder whether miR-H6 has different functions compared to other miRNAs (miR-H2-miR-H5) in HSV-1 infection. We therefore investigated the effects of miR-H6 targeting of ICP4 on HSV-1 replication, in particular, the effects on IL-6 production in human cornea cells. We found that miR-H6 targeting of ICP4 suppresses HSV-1 replication and decreases IL- 6 production in HCE, and this may provide an approach to prevent HSV-1 lytic infection and inhibit inflammation in cornea.

\section{Materials and Methods}

2.1. Cells and Virus Infection. HCE used in this study was derived from human limbal cells as described previously
$[33,34]$. Cells were cultured in DMEM/high-glucose supplemented with $10 \%$ fetal bovine serum (FBS; Gibco, NY, USA), $10 \mathrm{ng} / \mathrm{mL}$ human epidermal growth factor (EGF; Sigma, St. Louis, MO), $5 \mu \mathrm{g} / \mathrm{mL}$ human transferrin (Sigma), $5 \mu \mathrm{g} / \mathrm{mL}$ insulin, and $0.4 \mu \mathrm{g} / \mathrm{mL}$ hydrocortisone (Gibco BRL, Grand Island, NY). HEp-2 cells were grown in DMEM/F12 with $10 \%$ newborn bovine serum (Gibco, NY, USA). The cells were incubated at $37^{\circ} \mathrm{C}$ in a $5 \% \mathrm{CO}_{2}-95 \%$ air incubator. Stocks of the HSV-1 (F strain) were propagated on HEp-2 cells, and the titer of virus stocks was determined according to a previously described method [35].

HCE cells were infected by HSV-1 using a previously described method [5, 34]. Briefly, cells were cultured to 80 to $90 \%$ confluence, then infected with HSV-1 for $1 \mathrm{~h}$, with gentle $15 \mathrm{sec}$ shaking every $15 \mathrm{~min}$ to allow viral absorption. After $1 \mathrm{~h}$, the inoculum was removed and the medium was replaced with serum-free DMED/high-glucose. Cells infected at a multiplicity of infection (MOI) of $5,1,0.1$, were washed with phosphate-buffered saline (PBS) three times and harvested.

2.2. HSV-1 miR-H6 Mimics and Transfection. The miR-H6 mimics we used were double-stranded, chemically synthesized by Guangzhou RiboBio Co., Ltd. (Guangzhou, China) according to the mature miR-H6 (5'-CACUUCCCGUCCUUCCAUCCC- $3^{\prime}$ ). The mock is a small RNA that does not target any known gene as a negative control. The HCE cells were grown to $70-80 \%$ confluence and then transfected with miR-H6 (50 or $100 \mathrm{nM}$ ) and mock, by Lipofectamine 2000 (Invitrogen, Carlsbad, CA, USA), according to the manufacturer's instructions. Then, the cells are infected with HSV1 at MOI $0.124 \mathrm{~h}$ posttransfection. At the indicated time, the cells and medium were harvested for further experiments.

2.3. Plaque Assays. The plaque assay was performed as we described previously, but with modification [5]. HCE cells were grown in 12-well plates to 70 to $80 \%$ confluence and then transfected with miR-H6 mimics and infected with HSV-1 as described above. After absorbing the HSV-1 for $1 \mathrm{~h}$, cells were overlaid with $1 \mathrm{~mL}$ of a $1: 1$ mixture of low-melting-temperature agarose (NuSieve GTG Agarose, USA) and $2 \times$ DMEM/high glucose to permit only cell-to-cell spread of virus. At $48 \mathrm{~h}$ p.i., agarose was removed carefully and plates were stained with crystal violet for $20 \mathrm{~min}$ and then photographed. Finally, plaque sizes were measured.

2.4. Real-Time PCR Analysis. HCE cells were cultured to 80 to $90 \%$ confluence and then infected with HSV-1 in a multiplicity of infection (MOI) of 5, 1, and 0.1. At $24 \mathrm{~h}$ p.i., cells were washed with PBS three times and harvested. Cellular total RNA was isolated with TRIzol reagent (Invitrogen, Carlsbad, CA, USA) according to the manufacturer's instructions. Subsequently, RNA samples were detected using an All-in-One miRNA Q-PCR Detection kit (FulenGen, Guangzhou, China). The miR-H6 5' primers were CACTTCCCGTCCTTCCATCCCA (product size is 74) and homo snRNA U6 $5^{\prime}$ primers were CAAATTCGTGAAGCGTTCCATAT (product size is 79). The reaction conditions were 
$95^{\circ} \mathrm{C}$ for $10 \mathrm{~min}, 38$ cycles of $95^{\circ} \mathrm{C}$ for $10 \mathrm{~s}, 65^{\circ} \mathrm{C}$ for $20 \mathrm{~s}$, and $72^{\circ} \mathrm{C}$ for $10 \mathrm{~s}$ and $72^{\circ} \mathrm{C}$ for $5 \mathrm{~min}$ by iQ5 Real-Time PCR Detection System (Bio-Rad). The amount of each mRNA was calculated relative to the amount of U6 mRNA in the same samples by iQ software. Each run was completed with a melting curve analysis to confirm the specificity of the amplification.

2.5. Indirect Immunofluorescence. At 12 and $24 \mathrm{~h}$ p.i., slidemounted cells were used for indirect immunofluorescence analysis according to the method described previously [5]. Cells were incubated with mouse anti-human monoclonal antibody that recognizes HSV-1 ICP4 (Abcam, Cambridge, UK) at $4^{\circ} \mathrm{C}$ overnight. Cells were then incubated with TRITC-conjugated secondary goat anti-mouse IgG antibody (Zhongshan Goldenbridge, Beijing, China) at $37^{\circ} \mathrm{C}$ for $1 \mathrm{~h}$. The nuclei were stained with hoechst for $10 \mathrm{~min}$. Cells were then observed using a confocal laser scanning microscope (Carl Zeiss, Jena, Germany). Cells incubated with PBS (instead of the first antibody) were used as negative controls.

2.6. Western Blot Analysis. At $24 \mathrm{~h}$ p.i., cells were lysed with lysate buffer (20 nM tris-HCL). The samples were freezethawed 3 times and then centrifuged at $12,000 \mathrm{rpm}$ for $30 \mathrm{~min}$ at $4^{\circ} \mathrm{C}$ to remove cellular debris. Protein content in the supernatant was determined by the bicinchoninic acid method using BSA as the standard. Western blot was conducted according to our previous method [5]. The membranes were incubated with $1 \mu \mathrm{g} / \mathrm{mL}$ of mouse anti-human monoclonal antibodies that recognizes HSV-1 ICP4 (Abcam, Cambridge, UK) or $0.2 \mu \mathrm{g} / \mathrm{mL}$ mouse anti-GAPDH (KangChen, Shanghai, China) separately at $4^{\circ} \mathrm{C}$ overnight. Then, they were exposed to a secondary goat anti-mouse IgG antibody (Zhongshan Goldenbridge, Beijing, China) for $1 \mathrm{~h}$. Protein bands were visualized with a kit of chemiluminescence Phototope (R)-HRP Western Blot Detection System (Cell Signaling Technology, Inc., Danvers, MA, USA) and exposed by Kodak Imaging Station 4000MM (Kodak, Rochester, NY, USA).

2.7. Enzyme-Liked Immunosorbent Assay (ELISA). The HCE cells were transfected with miR-H6 and mock and then infected with HSV-1 as described above, or directly cultured the transfected cells without virus infection. The medium was collected at 6,12 , and $24 \mathrm{~h}$ p.i., or 30,36 , and $48 \mathrm{~h}$ post transfection for cells without HSV-1 infection, and the IL-6 levels in supernatant were determined using a specific IL-6 ELISA kit (Boster, Wuhan, China). Human IL-6 was used to construct a standard curve according to the manufacturer's instructions.

2.8. Statistical Analysis. All experiments were repetitively conducted a minimum of three times, and the quantitative data was expressed as means \pm SD. Statistical analysis of data was performed by one-way ANOVA (SPSS 17.0), with $P<0.05$ considered statistically significant.

\section{Results}

3.1. miR-H6 Effects on Cells and Viral Replication. HCE cells were transfected and infected HSV-1 at MOI 0.1 as described above, and cellular morphological changes were observed at 6,12 , and $24 \mathrm{~h}$ p.i. under phase-contrast microscopy (Figure $1(\mathrm{a})$ ). At $6 \mathrm{~h}$ p.i., there were no obvious differences in cellular morphology among cells transfected with miR-H6, the mock, without small RNA, and control cells (data not shown). At $12 \mathrm{~h}$ p.i., the cytopathic effect (CPE) could be observed in the mock and without small RNA cells, but not in the cells transfected with miR-H6 and control. Infected cells usually displayed clusters, and many individual cells remained uninfected. At $24 \mathrm{~h}$ p.i., CPE increased dramatically, and many giant, multinucleated cells could be seen in the mock and without small RNA cells. However, fewer CPE could be seen in cells transfected with miR-H6, including multinucleated giant cells.

Plaque assay was used to detect the virus quantity in MOI 0.1 HSV-1-infected HCE cells and demonstrated that normal cell had no formation of plaque, and HSV-1 without small RNA resulted in the formation of a larger number of plaques, which was similar to the mock. However, treatment with miR-H6 significantly decreased the plaque sizes and numbers (Figure 1(b)) and had significant difference (Figure 1(c), $P<0.05)$. Meanwhile, miR-H6 was measured in HSV1 -infected HCE with MOI 5, 1, and 0.1 , at $24 \mathrm{~h}$ p.i. The result showed that miR-H6 increased significantly in HSV-1infected HCEs, and the expression of miR-H6 was consistent with the concentration of the infectious virus quantity (Figure $1(\mathrm{~d}), P<0.01)$. The uninfected HCE cells had no expression of miR-H6.

3.2. miR-H6 Suppresses ICP4 Protein Expression. First, the ICP4 protein expression was observed by indirect immunofluorescence at 12 and $24 \mathrm{~h}$ p.i. Following HSV-1 infection and up to $12 \mathrm{~h}$ p.i., there was very weak ICP4 staining in HCE cells transfected with mock, and without mall RNA, less ICP4 expression was noted in HCE cells transfected with miR-H6; ICP4 was not observed in normal HCE control cells (Figure 2(a)). At $24 \mathrm{~h}$ p.i., the ICP4 protein expression increased dramatically. Compared with the cells transfected with mock and cells without small RNA, the intensity of immunostaining for ICP4 was dramatically weaker in the HCE cells transfected with miR-H6 (Figure 2(b)).

The effect of miR-H6 on the ICP4 protein was also detected by Western blot using antibodies against ICP4 (Figure 2(c)). At $24 \mathrm{~h}$ p.i., the expression of ICP4 decreased in the lysate of HCE cells transfected with miR-H6, whereas the expression in small RNA untreated cells and in mock remained constant (Figure $2(\mathrm{~d}), P<0.05$ ). Similarly, there was no expression of ICP4 in normal HCE cells.

3.3. The Effect of miR-H6 on IL-6 Release. The IL-6 protein expression in the medium was determined by ELISA (Figure 3). At $6 \mathrm{~h}$ p.i., the IL-6 level in medium of HCE cells transfected with miR-H6 was lower than that with mock and without small RNA; however, there was no significant 

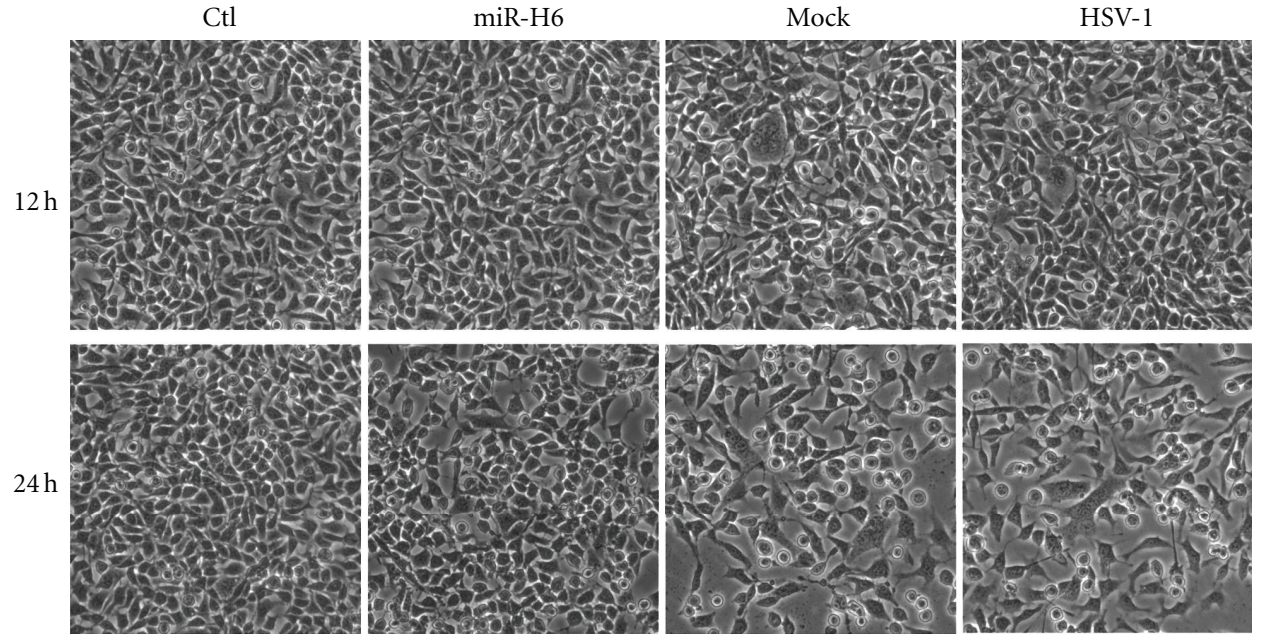

(a)
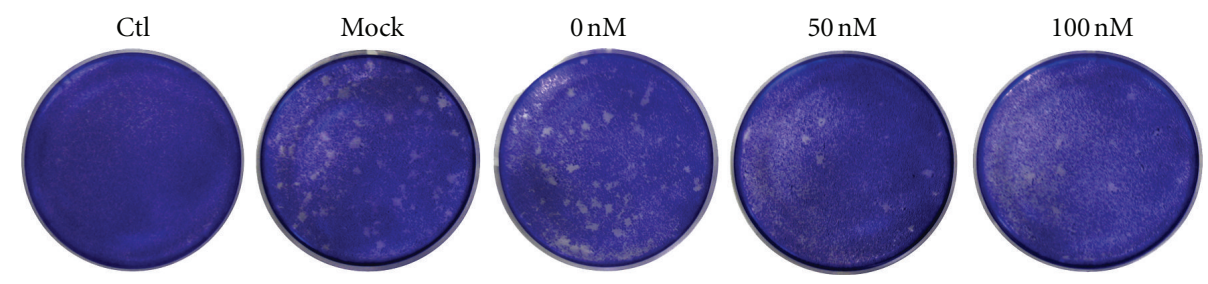

(b)

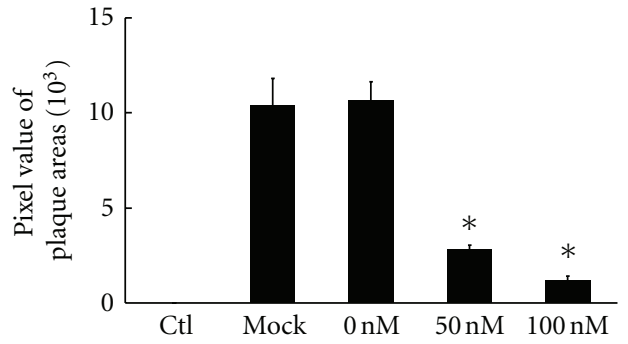

(c)

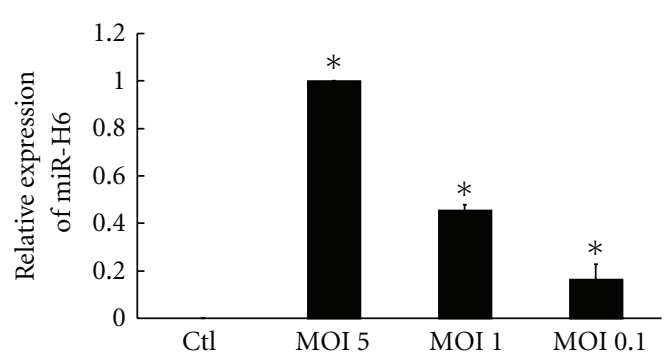

(d)

FIgURE 1: miR-H6 effects on cell changes and viral replication in HSV-1-infected HCE cells. HCE cells were transfected miR-H6 mimics and mock, followed by infection with MOI 0.1 of HSV-1 $24 \mathrm{~h}$ posttransfection. (a) The cellular morphological changes were imaged at $12 \mathrm{~h}$ and $24 \mathrm{~h}$ postinfection. CPE clearly increased, and many multinucleated giant cells could be seen in the cells treated with mock RNA and cells untreated with small RNA; fewer CPE and giant multinucleated cells could be seen in cells transfected with miR-H6 mimics. (b, c) Plaque assay showed that treatment with miR-H6 decreased the plaque sizes and numbers and had a statistically significant difference $(P<0.05)$. Cells were treated with miR-H6 mimics $(0,50,100 \mathrm{nM})$ and mock RNA (mock) or without small RNA (HSV-1). The control group (Ctl) consisted of cells without virus infection and small RNA treatment. (d) Real-time PCR determined miR-H6 expression in pooled HSV-1infected HCE cells, and miR-H6 levels were changed in a viral-titer-dependent manner $\left({ }^{*} P<0.01\right.$, one-way ANOVA, among MOI 0.1 , 1 , and 5).

difference $(P>0.05)$. At $12 \mathrm{~h}$ p.i., the IL-6 level in HCE cells transfected with miR-H6 was dramatically lower than in those transfected with mock or without small RNA (Figure 3(a), $P<0.01$ ). At $24 \mathrm{~h}$ p.i., the IL-6 levels in HCE transfected with mock and without small RNA decreased as compared to those at $12 \mathrm{~h}$ p.i. The IL- 6 level in medium of cells transfected with miR-H6 is insignificantly lower when compared to the level of mock transfection or that of only HSV-1-infected cells. The latter two showed significantly higher levels as compared to the control $(P<0.05)$.

The effects of miR-H6 on IL- 6 production of HCE cells were assayed by comparison of the IL-6 levels in HCE cells transfected with miR-H6 and mock and normal cells (Figure 3(b), $P>0.05$ ). IL-6 did not alter significantly among all the groups at various time points.

\section{Discussion}

During HSV-1 lytic infection, ICP4 upregulates the early and late genes, downregulates the IE genes through interaction with the transcription factors associated with RNA polymerase II, and represses the LAT promoter and prevents LAT production $[36,37]$. Also, ICP4 is the target of HSV-1 


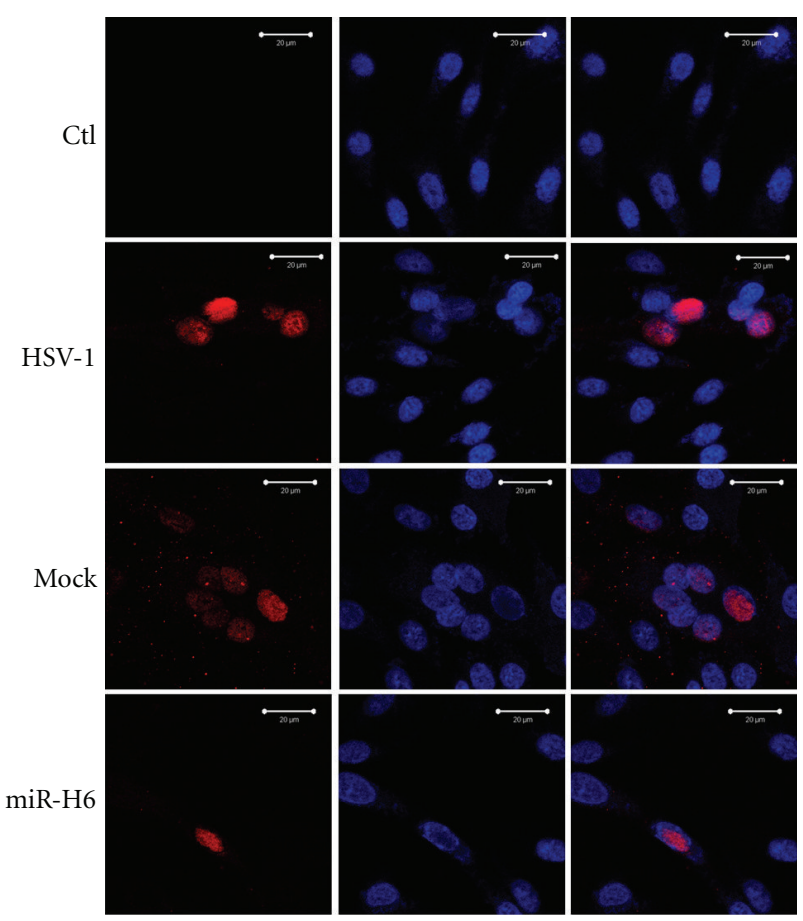

(a)

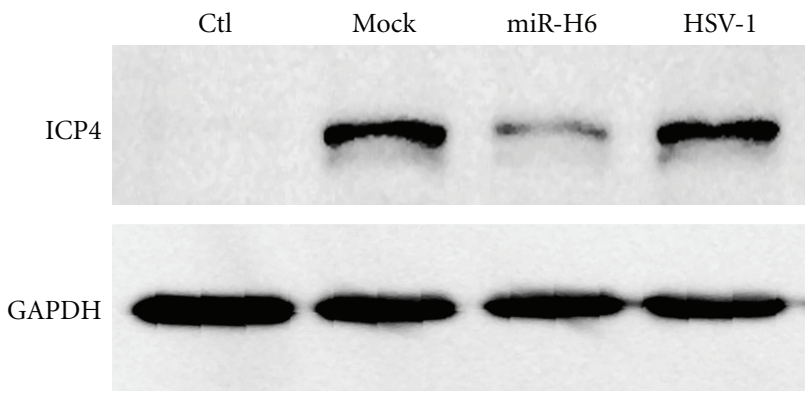

(c)

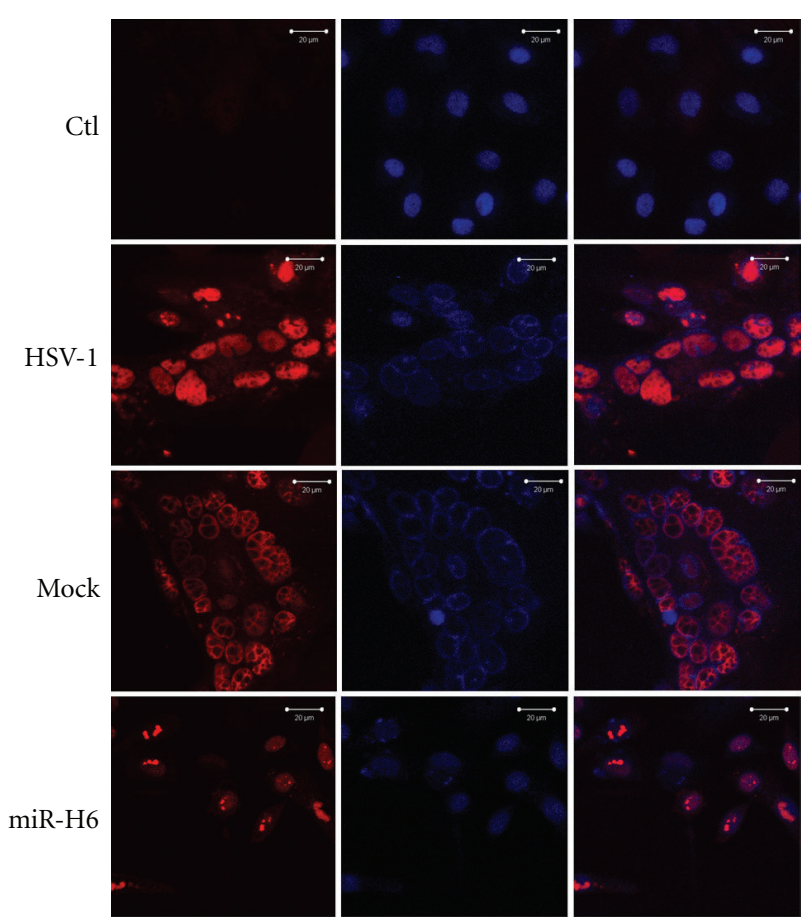

(b)

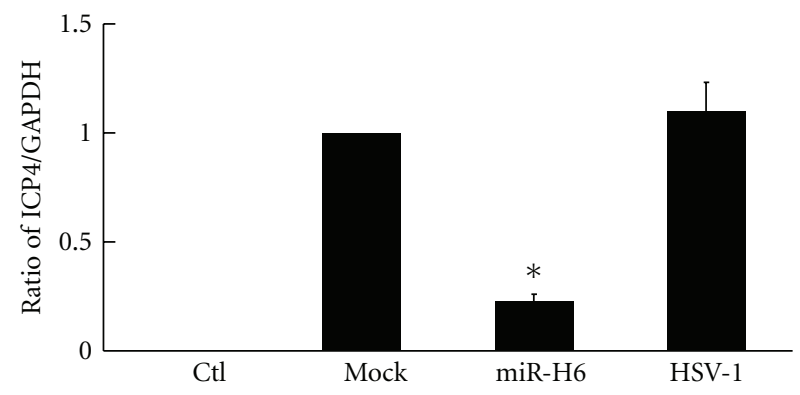

(d)

FIGURE 2: miR-H6 decreased ICP4 protein expression in HSV-1-infected HCE cells. HCE cells were transfected with miR-H6 mimics and mock RNA and then infected with MOI 0.1 of HSV-1 $24 \mathrm{~h}$ posttransfection. The staining of ICP4 protein in HCE cells at $12 \mathrm{~h}$ p.i. (a) and at 24 h p.i. (b) TRITC labeled the antibody-stained ICP4 (red, left) and the hoechst-dyed nucleus (blue, middle). Images of ICP4 and the nucleus were merged (right), scalebar: $20 \mu \mathrm{m}$. (c) The ICP4 band was determined by Western blot. (d) Quantitative analysis of bands in (c). Significantly lower levels of ICP4 in HCE cells transfected with miR-H6 ( ${ }^{*} P<0.05$, miR-H6 versus mock or HSV-1 alone).

miR-H6 [4]. Therefore, we began this study with the goal of observing the effect of miR-H6 on HSV-1 replication and the inflammatory cytokine IL- 6 production in HCE, by which HSV-1 infections can lead to cornea blindness [21,38]. We found that miR-H6 suppressed the expression of ICP4 protein and inhibited the HSV-1 productive infection in $\mathrm{HCE}$ and in human retinal pigment epithelial cells (data not shown). A similar result was reported by Umbach et al., who found that miR-H6 inhibits expression of ICP4 in 293T cells cotransfected with a synthetic miR-H6 duplex intermediate and with plasmids expressing either wild-type ICP4 or the ICP4 mutant [4]. Because viral miRNA regulation is dependent on the context of infection, such as cell-type and viral genome expression, we focused on the HSV-1-infected HCE to investigate miR-H6 roles on the productive infection.
The highly abundant expressions of microRNAs in HSV-1 latent infection were found in previous studies, which led to the concept that microRNA plays important roles in establishing and/or maintaining latent infections $[4,16,39]$. However, by experiments in acute and latent ganglionic infection in mice and lytic infection in Vero cells infected with wide-type or LAT deletion mutant HSV-1, Kramer et al. found that LAT deletion mutants establish and maintain latent infections and concluded that microRNAs are not essential for latency in mouse trigeminal ganglia, but may help promote it [17]. Du and colleagues found that the expression of viral genes in explanted ganglia was disordered rather than sequentially ordered as in infected cells in culture, and the accumulation of viral mRNAs takes place concurrently with a decrease in the accumulation of miRNAs/LATs, 


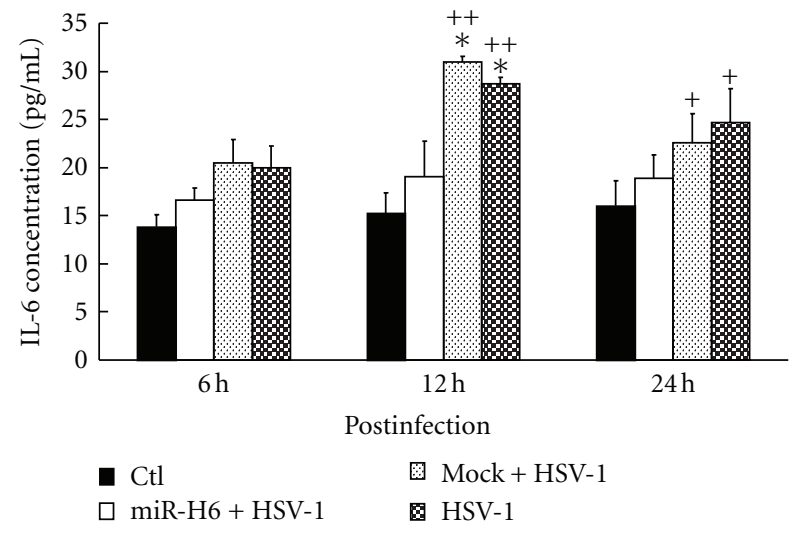

(a)

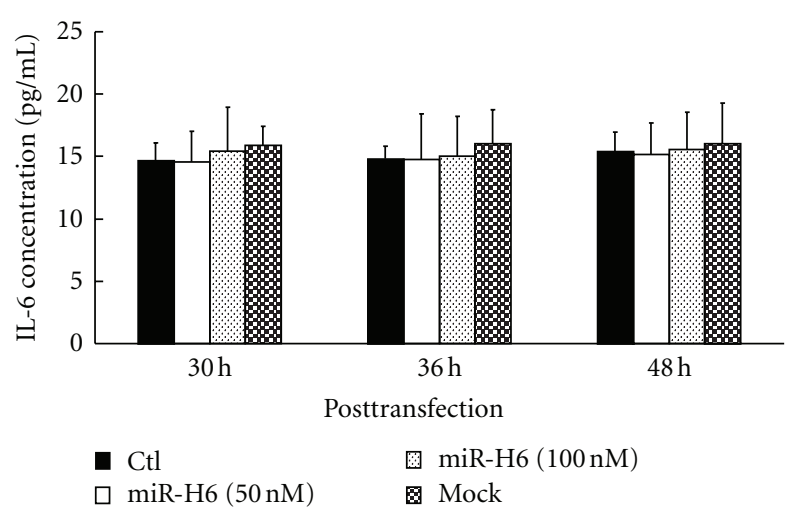

(b)

Figure 3: The effect of miR-H6 on IL-6 release in medium. HCE cells were transfected with miR-H6 and infected with MOI 0.1 of HSV1 after $24 \mathrm{~h}$ posttransfection (a) or without viral infection (b). The IL-6 level in medium was measured at indicated time p.i. by ELISA. * Statistical difference between HCE cells transfected with miR-H6 mimics and mock or HSV-1 alone $(P<0.01) ;{ }^{+/++}$statistical difference between normal HCE cells and mock or HSV-1 alone $\left({ }^{+} P<0.05 ;{ }^{++} P<0.01\right)$.

which was degraded by a viral gene product other than the short half-life of these miRNAs/LATs [40]. Nevertheless, studies on quantities of HSV-1 mircroRNAs revealed that miR-H6 and miR-H1 in the productive infection were the most abundant, whereas miR- $\mathrm{H} 2,-\mathrm{H} 3$, and $-\mathrm{H} 4$ were greatly expressed in the latent samples $[4,16,17]$. We also revealed that the expression of miR-H6 in HSV-1-infected HCE cells increases with viral concentration. All these data suggest that HSV-1 microRNAs may have stage-specific functions in the virus life cycle and that highly abundant levels of miR-H6 may have other roles in lytic infection other than maintaining the latent infection [17]. In our present study, we are interested in miR-H6 silencing ICP4 by transfecting synthesized miR-H6 mimics into HCE cells and followed by HSV-1 infection. More studies on miR-H6, such as interference with its function, and precise analysis of timedependent effects in lytic infection would have a greater effect on our understanding of the virus and its induced diseases.

HSV-1 infection in cornea is characterized by recurrent viral lytic infection, immune inflammation, neovascularisation, and so on. IL-6, as an important cytokine, targets multiple cell types and induces a broad array of responses. These responses are often, simplistically, classified as pro-or anti-inflammatory in nature [41]. It was showed that two major signal transduction pathways regulated by IL-6: STAT3 and SHP2/Gab/MAPK signaling, which involve the gp130 YxxQ and Y759 motives, respectively [42]. IL-6 plays important roles in these corneal pathological changes, which has been evidenced by previous studies [38, 43-46]. Also, HSV-1 infection influencing IL-6 has been previously reported. By using HSV-1 mutants lacking the virion-transactivating protein VP16 or any of ICP0, ICP4, ICP8, or ICP27, virusinfected leukocytes or murine epithelial cell line EMT-6 displayed unaltered capacities to induce IL-6 $[29,30]$. Thus any one of them alone is not essential for IL-6 production. It was reported recently that in Kaposi's sarcoma-associatedherpes-virus-(KSHV-) infected cells, miR-1293, targeting viral IL-6 RNA, and miR-608, targeting human IL-6 ORF, can mechanistically compete with KSHV ORF57 protein, which binds RNA export factor (REF) at the same site and in a similar way to ICP27, and thus influence IL-6 production $[47,48]$. Although in the present study we did not know if there was a target sequence of miR-H6 in HCE, we did not find miR-H6 suppressing IL-6 release in HCE without HSV-1 infection. Therefore, the miR-H6 decreasing IL-6 production in HSV-1-infected HCE cells is due to the suppression of virus production through inhibiting ICP4, because a functional viral genome was required for induction of IL-6 [29].

Our findings are that miR-H6 mimics could inhibit HSV1 productive infection in HCE cells as well as accompanied IL-6 release. Silencing viral microRNA targets as a novel antiviral therapy, our result may imply that in diseased tissue like keratitis, using miR-H6 could not only suppress viral replication, but also decrease inflammation due to IL-6 production.

\section{Conflict of Interests}

There is no commercial conflict of interests or any other conflicts of interests in this paper.

\section{Acknowledgments}

This work was supported by Grants from the National Natural Science Foundation of China (no. 81070765) and the Department of Science \& Technology of Guangdong Province, China (no. 10151008901000143).

\section{References}

[1] T. J. Taylor, M. A. Brockman, E. E. McNamee, and D. M. Knipe, “Herpes simplex virus," Front Biosci, vol. 7, pp. 752-764, 2002. 
[2] H. S. Toma, A. T. Murina, R. G. Areaux et al., "Ocular HSV-1 latency, reactivation and recurrent disease," Seminars in Ophthalmology, vol. 23, no. 4, pp. 249-273, 2008.

[3] N. Mador, D. Goldenberg, O. Cohen, A. Panet, and I. Steiner, "Herpes simplex virus type 1 latency-associated transcripts suppress viral replication and reduce immediate-early gene mRNA levels in a neuronal cell line," Journal of Virology, vol. 72, no. 6, pp. 5067-5075, 1998.

[4] J. L. Umbach, M. F. Kramer, I. Jurak, H. W. Karnowski, D. M. Coen, and B. R. Cullen, "MicroRNAs expressed by herpes simplex virus 1 during latent infection regulate viral mRNAs," Nature, vol. 454, no. 7205, pp. 780-783, 2008.

[5] F. Duan, S. Ni, Y. Nie, Q. Huang, and K. Wu, "SiRNA targeting for ICP4 inhibits HSV-1 replication in retinal pigment epithelial cells," Clinical and Experimental Ophthalmology, vol. 40, no. 2, pp. 195-204, 2012.

[6] C. Clusel, S. Meguenni, I. Elias, M. Vasseur, and M. Blumenfeld, "Inhibition of HSV-1 proliferation by decoy phosphodiester oligonucleotides containing ICP4 recognition sequences," Gene Expression, vol. 4, no. 6, pp. 301-309, 1995.

[7] Y. T. Liu, B. Song, Y. L. Wang et al., "SiRNA targeting ICP4 attenuates HSV-1 replication," Bing Du Xue Bao, vol. 26, no. 3, pp. 163-169, 2010.

[8] C. Y. Lee, P. S. Rennie, and W. W. Jia, "MicroRNA regulation of oncolytic herpes simplex virus-1 for selective killing of prostate cancer cells," Clinical Cancer Research, vol. 15, no. 16, pp. 5126-5135, 2009.

[9] D. P. Bartel, "MicroRNAs: genomics, biogenesis, mechanism, and function," Cell, vol. 116, no. 2, pp. 281-297, 2004.

[10] S. Pfeffer, M. Zavolan, F. A. Grässer et al., "Identification of virus-encoded microRNAs," Science, vol. 304, no. 5671, pp. 734-736, 2004.

[11] C. S. Sullivan and D. Ganem, "MicroRNAs and viral infection," Molecular Cell, vol. 20, no. 1, pp. 3-7, 2005.

[12] K. Takane and A. Kanai, "Vertebrate virus-encoded microRNAs and their sequence conservation," Japanese Journal of Infectious Diseases, vol. 64, no. 5, pp. 357-366, 2011.

[13] I. W. Boss and R. Renne, "Viral miRNAs: tools for immune evasion," Current Opinion in Microbiology, vol. 13, no. 4, pp. 540-545, 2010.

[14] C. Cui, A. Griffiths, G. Li et al., "Prediction and identification of herpes simplex virus 1-encoded microRNAs," Journal of Virology, vol. 80, no. 11, pp. 5499-5508, 2006.

[15] J. M. Hill, Y. Zhao, C. Clement, D. M. Neumann, and W. J. Lukiw, "HSV-1 infection of human brain cells induces miRNA-146a and Alzheimer-type inflammatory signaling," NeuroReport, vol. 20, no. 16, pp. 1500-1505, 2009.

[16] I. Jurak, M. F. Kramer, J. C. Mellor et al., "Numerous conserved and divergent microRNAs expressed by herpes simplex viruses 1 and 2," Journal of Virology, vol. 84, no. 9, pp. 4659-4672, 2010.

[17] M. F. Kramer, I. Jurak, J. M. Pesola, S. Boissel, D. M. Knipe, and D. M. Coen, "Herpes simplex virus 1 microRNAs expressed abundantly during latent infection are not essential for latency in mouse trigeminal ganglia," Virology, vol. 417, no. 2, pp. 239247, 2011.

[18] S. Tang, A. S. Bertke, A. Patel, T. P. Margolis, and P. R. Krause, "Herpes simplex virus 2 MicroRNA miR-H6 is a novel latencyassociated transcript-associated MicroRNA, but reduction of its expression does not influence the establishment of viral latency or the recurrence phenotype," Journal of Virology, vol. 85, no. 9, pp. 4501-4509, 2011.

[19] B. Roizman and R. J. Whitley, "The nine ages of herpes simplex virus," Herpes, vol. 8, no. 1, pp. 23-27, 2001.
[20] R. J. Whitley and F. Lakeman, "Herpes simplex virus infections of the central nervous system: therapeutic and diagnostic considerations," Clinical Infectious Diseases, vol. 20, no. 2, pp. 414-420, 1995.

[21] L. Remeijer, R. Duan, J. M. van Dun, M. A. W. Bettink, A. D. Osterhaus, and G. M. Verjans, "Prevalence and clinical consequences of herpes simplex virus type 1 DNA in human cornea tissues," Journal of Infectious Diseases, vol. 200, no. 1, pp. 1119, 2009.

[22] D. P. Kennedy, C. Clement, R. L. Arceneaux, P. S. Bhattacharjee, T. S. Huq, and J. M. Hill, "Ocular herpes simplex virus type 1 : is the cornea a reservoir for viral latency or a fast pit stop?" Cornea, vol. 30, no. 3, pp. 251-259, 2011.

[23] S. J. Divito and R. L. Hendricks, "Activated inflammatory infiltrate in HSV-1-infected corneas without herpes stromal keratitis," Investigative Ophthalmology and Visual Science, vol. 49, no. 4, pp. 1488-1495, 2008.

[24] K. R. Mott, C. J. Bresee, S. J. Allen, L. BenMohamed, S. L. Wechsler, and H. Ghiasi, "Level of herpes simplex virus type 1 latency correlates with severity of corneal scarring and exhaustion of $\mathrm{CD}^{+} \mathrm{T}$ cells in trigeminal ganglia of latently infected mice," Journal of Virology, vol. 83, no. 5, pp. 2246-2254, 2009.

[25] T. H. Stumpf, R. Case, C. Shimeld, D. L. Easty, and T. J. Hill, "Primary herpes simplex virus type 1 infection of the eye triggers similar immune responses in the cornea and the skin of the eyelids," Journal of General Virology, vol. 83, no. 7, pp. 1579-1590, 2002.

[26] D. M. Koelle, S. N. Reymond, H. Chen et al., "Tegument-specific, virus-reactive CD4 T cells localize to the cornea in herpes simplex virus interstitial keratitis in humans," Journal of Viro$\log y$, vol. 74, no. 23, pp. 10930-10938, 2000.

[27] P. C. Heinrich, I. Behrmann, G. Muller-Newen, F. Schaper, and L. Graeve, "Interleukin-6-type cytokine signalling through the gp130/Jak/STAT pathway," Biochemical Journal, vol. 334, no. 2, pp. 297-314, 1998.

[28] J. D. Kriesel, J. Ricigliano, S. L. Spruance, H. H. Garza Jr., and J. M. Hill, "Neuronal reactivation of herpes simplex virus may involve interleukin-6," Journal of NeuroVirology, vol. 3, no. 6, pp. 441-448, 1997.

[29] S. R. Paludan, "Requirements for the induction of interleukin6 by herpes simplex virus-infected leukocytes," Journal of Virology, vol. 75, no. 17, pp. 8008-8015, 2001.

[30] S. Kanangat, J. S. Babu, D. M. Knipe, and B. T. Rouse, "HSV-1mediated modulation of cytokine gene expression in a permissive cell line: selective upregulation of IL-6 gene expression," Virology, vol. 219, no. 1, pp. 295-300, 1996.

[31] G. Donnarumma, V. De Gregorio, A. Fusco et al., "Inhibition of HSV-1 replication by laser diode-irradiation: possible mechanism of action," International Journal of Immunopathology and Pharmacology, vol. 23, no. 4, pp. 1167-1176, 2010.

[32] K. Hayashi, L. C. Hooper, M. S. Chin, C. N. Nagineni, B. Detrick, and J. J. Hooks, "Herpes simplex virus 1 (HSV-1) DNA and immune complex (HSV-1-human IgG) elicit vigorous interleukin 6 release from infected corneal cells via Toll-like receptors," Journal of General Virology, vol. 87, no. 8, pp. 2161-2169, 2006.

[33] J. liu, G. Song, Z. Wang et al., "Establishment of a corneal epithelial cell line spontaneously derived from human limbal cells," Experimental Eye Research, vol. 84, no. 3, pp. 599-609, 2007.

[34] Y. Nie, D. Cui, Z. Pan, J. Deng, Q. Huang, and K. Wu, "HSV-1 infection suppresses TGF- $\beta 1$ and SMAD3 expression in human corneal epithelial cells," Molecular Vision, vol. 14, pp. 1631-1638, 2008. 
[35] W. K. Baumgaertner, A. E. Metzler, S. Krakowa, and A. Koestner, "In vitro identification and characterization of a virus isolated from a dog with neurological dysfunction," Infection and Immunity, vol. 31, no. 3, pp. 1177-1183, 1981.

[36] N. A. DeLuca and P. A. Schaffer, "Activation of immediateearly, early, and late promoters by temperature-sensitive and wild-type forms of herpes simplex virus type 1 protein ICP4," Molecular and Cellular Biology, vol. 5, no. 8, pp. 1997-2008, 1985.

[37] C. A. Smith, P. Bates, R. Rivera-Gonzalez, B. Gu, and N. A. DeLuca, "ICP4, the major transcriptional regulatory protein of herpes simplex virus type 1 , forms a tripartite complex with TATA-binding protein and TFIIB," Journal of Virology, vol. 67, no. 8, pp. 4676-4687, 1993.

[38] L. J. Al-Dujaili, P. P. Clerkin, C. Clement et al., "Ocular herpes simplex virus: how are latency, reactivation, recurrent disease and therapy interrelated?" Future Microbiology, vol. 6, no. 8, pp. 877-907, 2011.

[39] J. L. Umbach, M. A. Nagel, R. J. Cohrs, D. H. Gilden, and B. R. Cullen, "Analysis of human alphaherpesvirus microRNA expression in latently infected human trigeminal ganglia," Journal of Virology, vol. 83, no. 20, pp. 10677-10683, 2009.

[40] T. Du, G. Zhou, and B. Roizman, "HSV-1 gene expression from reactivated ganglia is disordered and concurrent with suppression of latency-associated transcript and miRNAs," Proceedings of the National Academy of Sciences of the United States of America, vol. 108, no. 46, pp. 18820-18824, 2011.

[41] R. S. Jawa, S. Anillo, K. Huntoon, H. Baumann, and M. Kulaylat, "Analytic review: interleukin-6 in surgery, trauma, and critical care: part I: basic science," Journal of Intensive Care Medicine, vol. 26, no. 1, pp. 3-12, 2011.

[42] T. Hirano, "Interleukin 6 in autoimmune and inflammatory diseases: a personal memoir," Proceedings of the Japan Academy Series B, vol. 86, no. 7, pp. 717-730, 2010.

[43] H. Li, J. Zhang, A. Kumar, M. Zheng, S. S. Atherton, and F. S. $\mathrm{X}$. Yu, "Herpes simplex virus 1 infection induces the expression of proinflammatory cytokines, interferons and TLR7 in human corneal epithelial cells," Immunology, vol. 117, no. 2, pp. 167-176, 2006.

[44] J. D. Kriesel, B. M. Gebhardt, J. M. Hill et al., "Antiinterleukin-6 antibodies inhibit herpes simplex virus reactivation," Journal of Infectious Diseases, vol. 175, no. 4, pp. 821827, 1997.

[45] V. Arrunategui-Correa, S. Baltatzis, and C. S. Foster, "The role of cytokines in experimental herpes simplex keratitis," Acta Virologica, vol. 43, no. 5, pp. 325-329, 1999.

[46] P. S. Biswas, K. Banerjee, P. R. Kinchington, and B. T. Rouse, "Involvement of IL-6 in the paracrine production of VEGF in ocular HSV-1 infection," Experimental Eye Research, vol. 82, no. 1, pp. 46-54, 2006.

[47] R. B. Tunnicliffe, G. M. Hautbergue, P. Kalra et al., "Structural basis for the recognition of cellular mRNA export factor REF by herpes viral proteins HSV-1 ICP27 and HVS ORF57," Plos Pathogens, vol. 7, no. 1, Article ID e1001244, 2011.

[48] J. G. Kang, N. Pripuzova, V. Majerciak, M. Kruhlak, S.-Y. Le, and Z. M. Zheng, "Kaposi's sarcoma-associated herpesvirus ORF57 promotes escape of viral and human interleukin-6 from microRNA-mediated suppression," Journal of Virology, vol. 85 , no. 6 , pp. 2620-2630, 2011. 


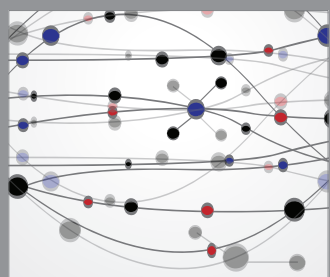

The Scientific World Journal
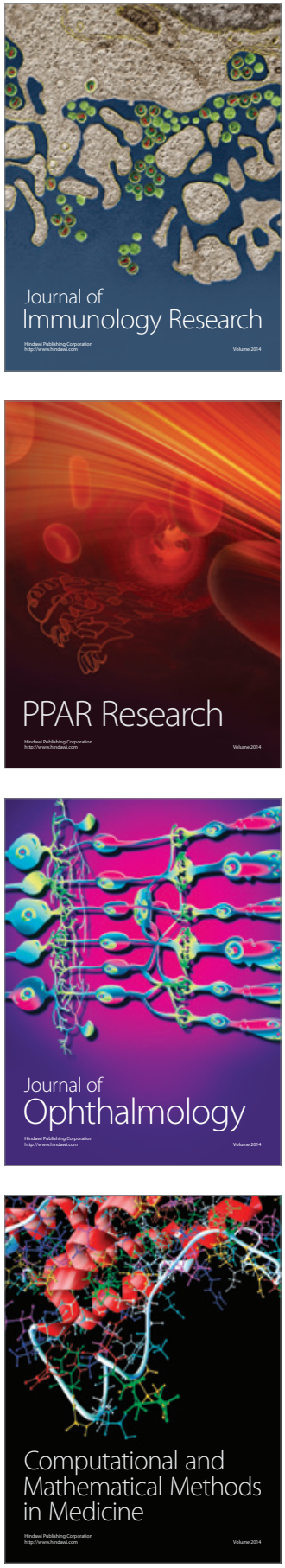

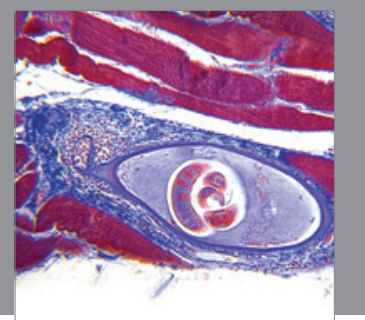

Gastroenterology

Research and Practice
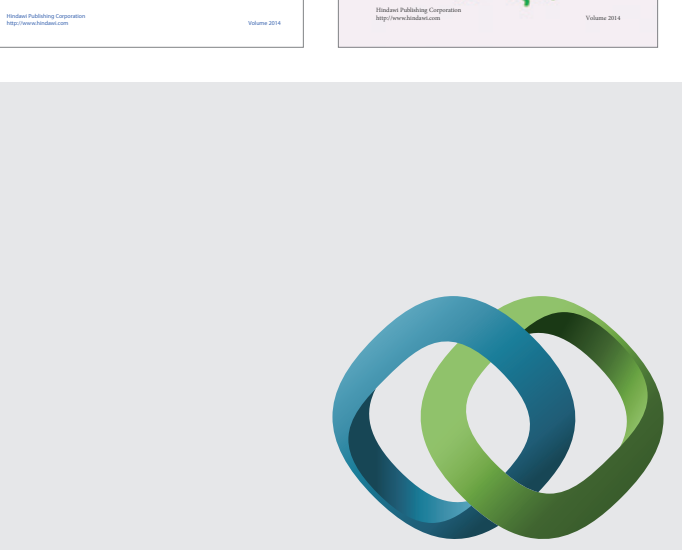

\section{Hindawi}

Submit your manuscripts at

http://www.hindawi.com
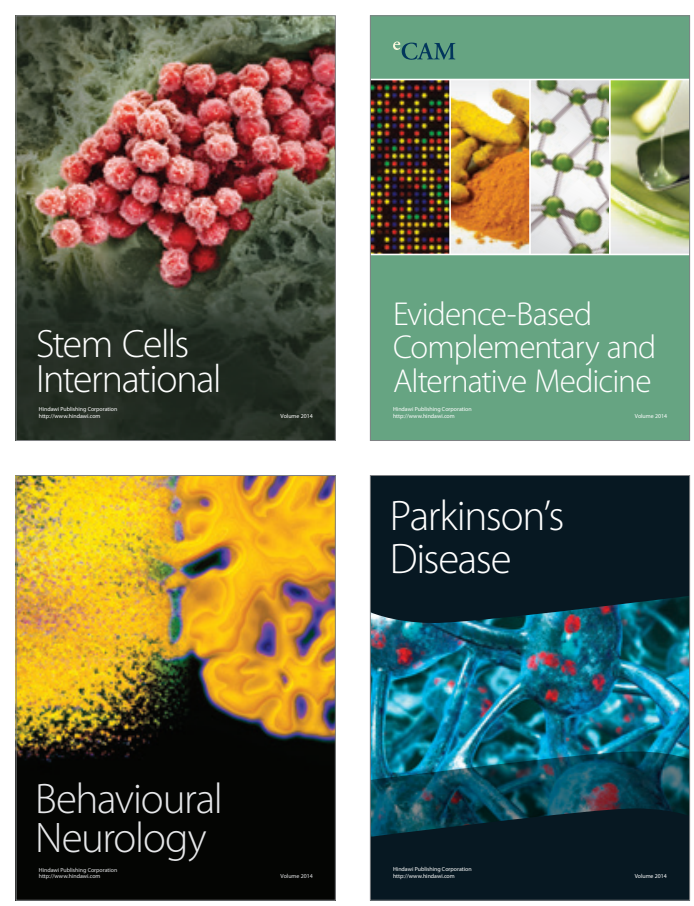

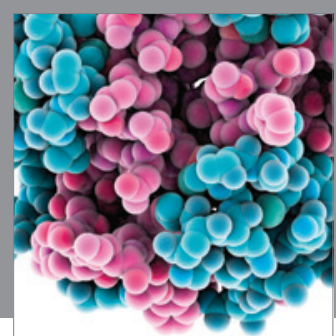

Journal of
Diabetes Research



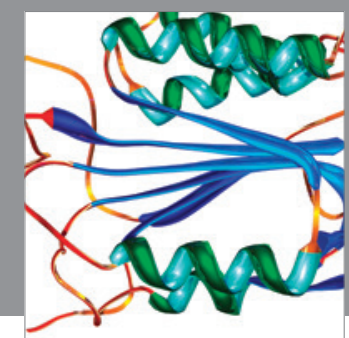

Disease Markers
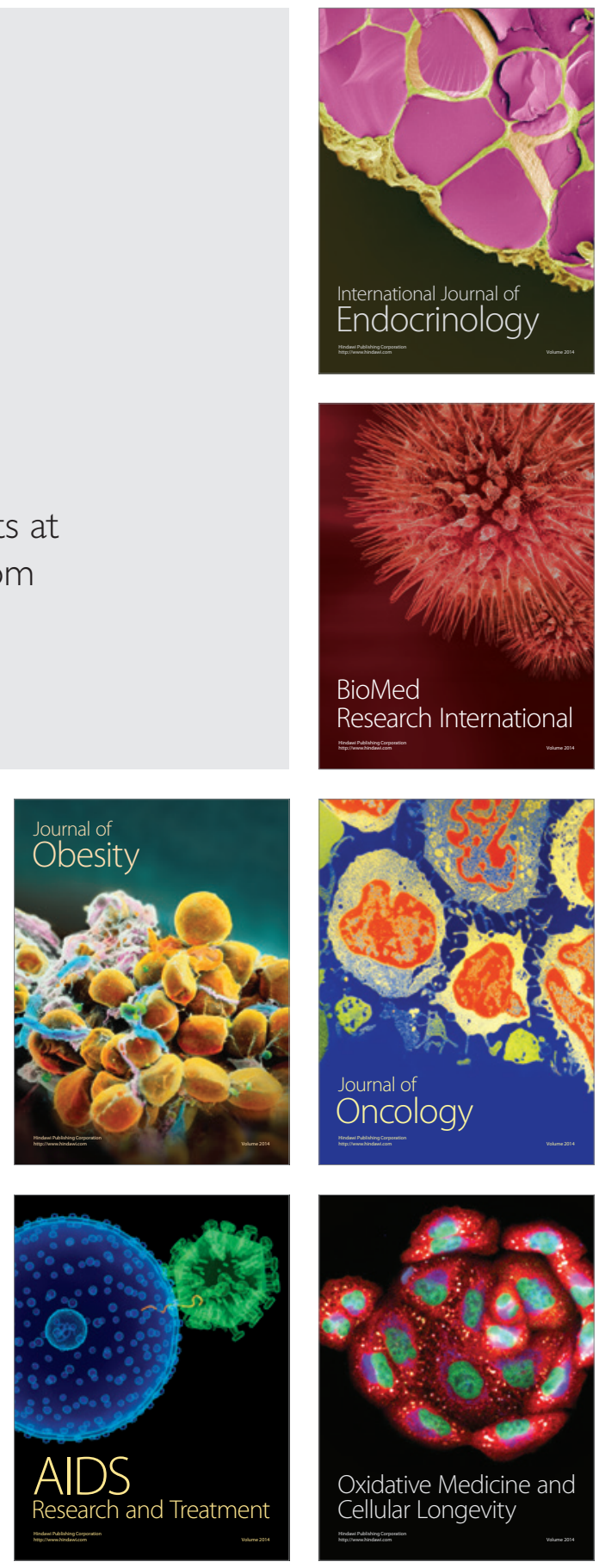